\title{
BMJ Open Organising Support for Carers of Stroke Survivors (OSCARSS): a cluster randomised controlled trial with economic evaluation
}

\author{
Emma Patchwood (D) ,,2 Kate Woodward-Nutt, ${ }^{2}$ Sarah A Rhodes, ${ }^{2,3}$ \\ Evridiki Batistatou, ${ }^{2,3}$ Elizabeth Camacho (D) , ${ }^{4}$ Sarah Knowles, ${ }^{2,5,6}$ \\ Sarah Darley (D) ,2,5 Gunn Grande (D) ,2,7 Gail Ewing (D) , 2,8 Audrey Bowen (D) 1,2
}

To cite: Patchwood E, Woodward-Nutt K, Rhodes SA, et al. Organising Support for Carers of Stroke Survivors (OSCARSS): a cluster randomised controlled trial with economic evaluation. BMJ Open 2021;11:e038777. doi:10.1136/ bmjopen-2020-038777

- Prepublication history and supplemental material for this paper is available online. To view these files, please visit the journal online (http://dx.doi. org/10.1136/bmjopen-2020038777).

Received 23 March 2020

Revised 10 December 2020

Accepted 14 December 2020

\section{SLinked}

http://dx.doi.org/10.1136/ bmjopen-2020-038129

\section{Check for updates}

(c) Author(s) (or their employer(s)) 2021. Re-use permitted under CC BY-NC. No commercial re-use. See rights and permissions. Published by BMJ.

For numbered affiliations see end of article.

\section{Correspondence to} Professor Audrey Bowen; audrey.bowen@manchester. ac.uk

\section{ABSTRACT}

Objective Investigated clinical effectiveness and costeffectiveness of a person-centred intervention for informal carers/caregivers of stroke survivors.

Design Pragmatic cluster randomised controlled trial (cRCT) with economic and process evaluation.

Setting Clusters were services, from a UK voluntary sector specialist provider, delivering support primarily in the homes of stroke survivors and informal carers. Participants Adult carers in participating clusters were referred to the study by cluster staff following initial support contact.

Interventions Intervention was the Carer Support Needs Assessment Tool for Stroke: a staff-facilitated, carerled approach to help identify, prioritise and address the specific support needs of carers. It required at least one face-to-face support contact dedicated to carers, with reviews as required. Control was usual care, which included carer support (unstructured and variable). Outcome measures Participants provided study entry and self-reported outcome data by postal questionnaires, 3 and 6 months after first contact by cluster staff. Primary outcome: 3-month caregiver strain (Family Appraisal of Caregiving Questionnaire, FACQ). Secondary outcomes: FACQ subscales of caregiver distress and positive appraisals of caregiving, mood (Hospital Anxiety and Depression Scale) and satisfaction with stroke services (Pound). The economic evaluation included self-reported healthcare utilisation, intervention costs and EQ-5D-5L. Randomisation and masking Clusters were recruited before randomisation to intervention or control, with stratification for size of service. Cluster staff could not be masked as training was required for participation. Carer research participants provided self-reported outcome data unaware of allocation; they consented to follow-up data collection only.

Results Between 1 February 2017 and 31 July 2018, 35 randomised clusters (18 intervention; 17 control) recruited 414 cRCT carers (208 intervention; 206 control). Study entry characteristics were well balanced. Primary outcome measure: intention-to-treat analysis for $84 \%$ retained participants (175 intervention; 174 control) found mean (SD) FACQ carer strain at 3 months to be $3.11(0.87)$ in the control group compared with $3.03(0.90)$ in the intervention group, adjusted mean difference of -0.04

\section{Strengths and limitations of this study}

- We successfully conducted the first adequately powered cluster randomised controlled trial of an approach to support informal carers of stroke survivors, but may have benefited from a feasibility trial to maximise intervention fidelity.

- We collaborated closely with service providers and previous service users to pragmatically tailor the intervention for implementation, including a stafftraining package.

- The demographic profile of the sample was as expected for carers of stroke survivors but the sample lacked ethnic diversity and we may have benefited from seeking data beyond 6 months after support had been initiated.

- We highlight the feasibility of robust research with this population and signpost to suggestions from our nested process evaluation for improved implementation of person-centred care.

$(95 \% \mathrm{Cl}-0.20$ to 0.13$)$. Secondary outcomes had similarly small differences and tight Cls. Sensitivity analyses suggested robust findings. Intervention fidelity was not achieved. Intervention-related group costs were marginally higher with no additional health benefit observed on EQ$5 \mathrm{D}-5 \mathrm{~L}$. No adverse events were related to the intervention. Conclusions The intervention was not fully implemented in this pragmatic trial. As delivered, it conferred no clinical benefits and is unlikely to be cost-effective compared with usual care from a stroke specialist provider organisation. It remains unclear how best to support carers of stroke survivors. To overcome the implementation challenges of person-centred care in carers' research and service development, staff training and organisational support would need to be enhanced.

Trial registration number ISRCTN58414120.

\section{INTRODUCTION}

Informal carers, providing unpaid support to family and friends with long-term health conditions, make an invaluable societal and 
economic contribution. $B M J$ published the 'unremitting burden on carers' over 30 years ago, ${ }^{1}$ but sadly carers' own support needs are still often overlooked and being a caregiver often adversely affects health and well-being. ${ }^{2}$

Although countries such as the UK now mandate for the identification of carers' support needs through the 2014 Care Act, ${ }^{3}$ less than one-third report receiving a statutory assessment. ${ }^{2}$ One possible approach for comprehensive support is the Carer Support Needs Assessment Tool (CSNAT) intervention. ${ }^{4}$ The CSNAT intervention has multiple components including a comprehensive assessment tool integrated within a staged carer-led approach to individualised support. It was developed, implemented and tested in the context of palliative care with positive outcomes, including a significant reduction in caregiver strain as measured on the Family Appraisal of Caregiving Questionnaire (FACQ).$^{5-9}$ We hypothesised that this intervention had the potential to support informal carers of people with long-term health conditions such as stroke, that causes a greater range of disabilities than any other in the UK. ${ }^{10}$ Recent systematic reviews and trials of carers of stroke survivors have highlighted the absence of a robustly proven support intervention. ${ }^{11-15}$

In close collaboration with a study-specific carer advisory research group (see the Patient and public involvement section) and a UK stroke service provider organisation, we adapted the CSNAT intervention including a staff training and implementation package tailored to the provider organisation (see the Interventions and procedures section, and figure 1 , table 1 and online supplemental table S1). This partnership was facilitated by the former National Institute for Health Research Collaboration for Leadership in Applied Health Research and Care Greater Manchester (NIHR CLAHRC GM, https://www. clahrc-gm.nihr.ac.uk/, now Applied Research Collaborations). The aim of the Organising Support for Carers of Stroke Survivors (OSCARSS) study was to determine the clinical and cost-effectiveness of the CSNAT-Stroke intervention for carers of stroke survivors, when compared with usual care. The primary hypothesis was that the

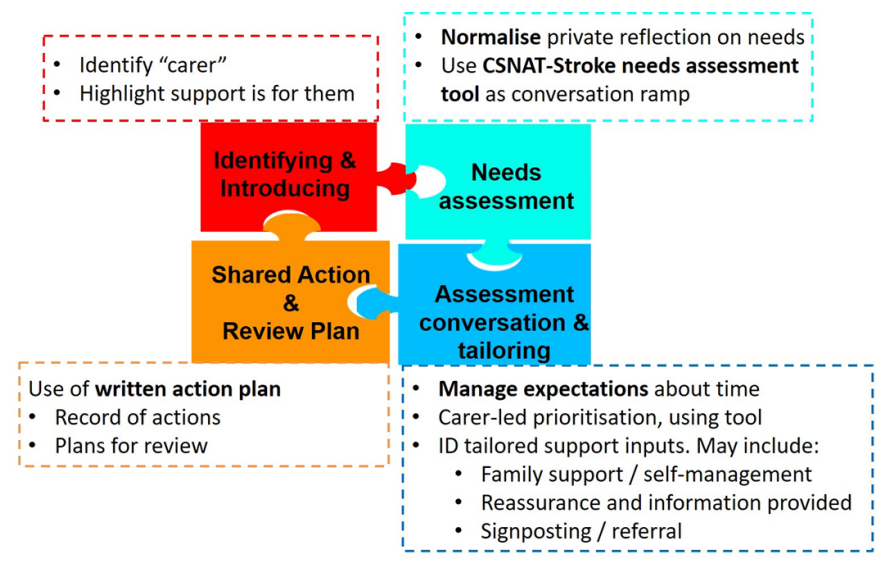

Figure 1 The adapted CSNAT-Stroke intervention as intended. CSNAT-Stroke, Carer Support Needs Assessment Tool for Stroke.
Table 1 Summary of key differences between intervention and usual care

\begin{tabular}{ll}
\hline Usual care & CSNAT-Stroke intervention \\
\hline $\begin{array}{l}\text { Focus primarily on stroke } \\
\text { survivor }\end{array}$ & Focus specifically on carer \\
$\begin{array}{l}\text { No formal process with } \\
\text { carers: varies across } \\
\text { services }\end{array}$ & $\begin{array}{l}\text { A standardised assessment and } \\
\text { support process for carers }\end{array}$ \\
$\begin{array}{ll}\text { Support carer if present } & \text { Make appointment to include } \\
\text { carer }\end{array}$ \\
$\begin{array}{ll}\text { Usually see together with } \\
\text { stroke survivor }\end{array}$ & $\begin{array}{l}\text { Normalise seeing carer } \\
\text { separately }\end{array}$ \\
$\begin{array}{l}\text { If carer present: prompt } \\
\text { question from practitioner } \\
\text { about carer's own needs }\end{array}$ & $\begin{array}{l}\text { Carer-led assessment and } \\
\text { prioritisation of needs using } \\
\text { evidence-based assessment } \\
\text { tool and staged person-centred } \\
\text { approach }\end{array}$ \\
\hline Review times vary & $\begin{array}{l}\text { Carer-specific action and review } \\
\text { plan }\end{array}$ \\
\hline
\end{tabular}

CSNAT-Stroke, Carer Support Needs Assessment Tool for Stroke.

adapted intervention would reduce caregiver strain when compared with usual care. Secondary hypotheses explored the impact on other aspects of the carer experience (eg, well-being and satisfaction with services), as well as its economic impact.

\section{METHODS}

\section{Study design}

OSCARSS was a longitudinal, pragmatic, national cluster randomised controlled trial (cRCT), underpinned by patient and public involvement from our study-specific carer advisory research group. ${ }^{16}$ Cluster randomisation was essential to avoid contamination. Clusters were drawn from services commissioned by the National Health Service (NHS) or local authorities, delivered by a UK voluntary sector stroke specialist organisation providing long-term support to stroke survivors and carers, including hospital and home visits. Eligible clusters were those with capacity for research participation and delivering support to carers in their own homes and a minimum of five new client (survivor or carer) referrals per month, based on historical service delivery records from a 9-month period before the study began.

This paper focuses on the RCT to explore the intervention's clinical and cost-effectiveness. OSCARSS also included a mixed-methods embedded process evaluation to help understand intervention implementation and workforce adoption, described in detail elsewhere. ${ }^{17}$

Ethics approvals were obtained (see Ethics approval section) and the lead author (EP) affirms that this manuscript is an honest, accurate and transparent account of the study being reported. The study methods and design have been described in detail, with no major changes made to protocol. ${ }^{18}$ 


\section{Patient and public involvement}

A study-specific Research User Group (RUG) of 10 individuals with experience of caring for a stroke survivor was set up in December 2015, at the planning stages of OSCARSS. Through regular meetings (2015-2019) and representation on the Trial Management Group, the priorities, experiences and preferences of the RUG informed development of the research questions and the design, analysis/interpretation and dissemination of all components of the OSCARSS Study. The RUG supported authorship of an easy access report on the results of this study that has been sent to study participants (Dissemination Declaration).

The RUG advised on participant recruitment and were central in limiting the burden of participation for carers. The RUG also were key in supporting adaptation of the research intervention (CSNAT-Stroke) and staff training package, including role-playing videos of the intervention in practice. A video summarising their role in OSCARSS is available on the study website: https://www.arc-gm. nihr.ac.uk/projects/oscarss, and following GRIPP2 framework ${ }^{19}$ we have published a separate paper on the working practices and experiences of RUG members and the researchers who facilitated the group meetings. ${ }^{16}$

\section{Participants}

English-speaking informal carers of stroke survivors were eligible if they were over 18 years old and received at least one face-to-face support contact from participating cluster staff. Carers could be included at any time poststroke event with any level of need or support requirements. We focused on those newly referred to the service as opposed to those using services for some time as core parts of the intervention included identification of carers. We aimed to recruit those individuals identified as 'primary caregiver', even when there may have been other informal carers involved.

Following the first face-to-face support contact (either intervention or control), eligible carers were invited by cluster staff to find out more about potential study participation and given up to 6 weeks to make a decision. Carers were assured that their decision on study participation would have no impact on the provision of ongoing support (either intervention or control). If carers accepted, their details were passed securely to the research team who provided full study information by post and sought informed written consent to participate. Procedures were also in place for consent to be taken by telephone. Researchers were in regular contact with all cluster staff and senior leadership to encourage fidelity with research procedures, including the consistent invitation to participate for all eligible carers.

\section{Randomisation and masking}

Details of the randomisation and masking were described in the protocol. ${ }^{18}$ Briefly, clusters were recruited (with consent of senior leadership and frontline staff within the provider organisation) by research staff before randomisation to ensure allocation concealment at a cluster level. Clusters were block randomised to intervention or control, with stratification for size of service using random blocks of two (to ensure similar numbers of carers and clusters in each arm). The trial statistician performed the randomisation of all recruited clusters simultaneously using an anonymised list of cluster ID numbers and size of service data. The initial randomisation list produced allocations for 36 clusters, with a second randomisation list produced to allocate up to 16 clusters in the event of needing to replace clusters that dropped out or failed to recruit.

Cluster staff could not be masked as training was required to equip them to participate in the study. Training included participant recruitment and trial procedures (control and intervention arms of the trial) and the intervention (intervention arm only). The research team were masked to allocation as far as possible, although some team members could become unmasked during cluster staff training or support activities. Carer research participants provided self-report primary and secondary outcomes unaware of allocation; they received support from their local randomised cluster and consented to follow-up data collection only. Carers were told that the service was being evaluated but not told about the randomised clusters.

\section{Interventions and procedures}

The intervention is a person-centred, structured process of assessment and support that is practitioner facilitated, but carer led. It enables carers to identify and prioritise their unmet needs during routine support contacts by staff; and then collaboratively put in place tailored support to meet identified needs. The intervention includes: a needs assessment tool; an action plan; and a multistage personcentred framework for introducing and using them both. The intervention is delivered typically at home visits that also include stroke survivors being supported by the same staff member. Staff in all clusters were trained in the study processes but only those in intervention clusters were trained to implement this individualised approach, using instructional videos, role-play and workbooks. Implementation does not include change to local, external support services available to carers-although staff were encouraged to create service directories, in case signposting or referral was required. The intended intervention is illustrated in figure 1 , summarised in table 1 which highlights differences to usual care and described in detail in online supplemental table S1, adapted Template for Intervention Description and Replication checklist. ${ }^{20}$

We compared the intervention to usual care within clusters (also summarised in table 1 and described in online supplemental table S1). Although the service delivery organisation had well-defined practices for supporting stroke survivors, support for carers was typically offered but variable across services.

Study entry data included demographic and clinical characteristics of carers and their cared-for stroke 
survivors, along with EQ-5D-5L. ${ }^{21}$ These were collected through carer self-report postal questionnaires at the same time as consent. As support (intervention or control) was implemented at a cluster level and designed to begin at the first point of contact with a carer, study entry data could not be considered truly 'baseline' as it was collected after support had been initiated, although data such as age, gender and date of stroke could be assumed to be constant. Initial and follow-up outcomes were sought by carer self-report postal questionnaire 3 and 6 months after support was initiated. In addition, service delivery records for all consented carers were extracted by the service provider at the end-of-study data collection.

\section{Outcomes}

Primary outcome was the strain subscale from the $F A C Q^{9}$ 3 months after the start of intervention. Three-month and 6-month outcomes postal questionnaire packs were identical in content. Carers were provided with the option to complete them over the telephone with support from a researcher. Packs included:

- The FACQ ${ }^{9}$ with subscales for strain, distress and positive appraisals of the impact of caring. Each item was scored from 1 to 5 and each subscale produced a mean score out of 5 , with a score of 3 as neutral, and higher scores indicating a greater amount of the variable being measured.

- The Pound Carer Satisfaction with Stroke Services Scale, ${ }^{22}$ with higher scores indicating more satisfaction with services (composite score maximum of 44; standalone 'smiley faces' overall score maximum of 7).

- The Hospital Anxiety and Depression Scale ${ }^{23}$ for carer anxiety and depression, with higher scores indicating higher mood disturbance and clinical cut-offs of: noncases (0-7); mild (8-10); moderate (11-14); severe (15-21).

- An adapted version of the Service Receipt Inventory ${ }^{11}$ to collect information on carers' use of NHS and social care services and the EQ-5D-5L $\mathrm{L}^{21}$ as the measure of health benefit used in the economic evaluation.

Routinely collected service delivery records for consented carers (described in Interventions and Procedures) included: the dates, types and duration of direct and non-direct support activities provided; standardised entries from staff pertaining to needs identified and actions taken during support contacts. Needs and action categories were pre-existing within the service provider records management system and not altered for the purpose of the trial.

We collected data on how often staff used the intervention's needs assessment tool and action plan but primarily evaluated implementation using qualitative methods in our separately reported process evaluation. ${ }^{17}$

No serious adverse events (SAEs) were expected to be related to the intervention. All known AEs were typically collected via outcomes postal packs or during routine study follow-up calls with participants or cluster staff. SAEs were reported if they were deemed related and unexpected. Protocol deviations were recorded, for example, return of 3-month outcome measures more than 6 weeks late.

\section{Statistical and economic analysis}

A full Statistical Analysis Plan was published with the study protocol. ${ }^{18}$ We explored a range of projected sample sizes in our protocol. A minimum of 400 carers recruited from 32 clusters (200 per trial arm) would provide $80 \%$ power to detect standardised effect sizes on the primary outcome of 0.31 or more (FACQ Strain mean score), assuming an intraclass correlation coefficient (ICC) of 0.01 with a $20 \%$ loss to follow-up, at the $5 \%$ significance level. Power was calculated using the Stata clsampsi function. ${ }^{24}$ We did not expect the cluster ICC to be $>0.05 .{ }^{25}$

The primary analysis was intention to treat (ITT), comparing intervention and control at 3 months using a multilevel regression model with adjustment for clustering and using the following fixed individual level covariates: time post-stroke; age of carer; health of carer at study entry; stroke severity (as rated by carer); and the following cluster level covariates: size of service and experience of staff delivering support. Missing covariate data were imputed using multiple imputation via the 'mi impute' function in Stata. Sequential imputation using chained equations was used to create 10 datasets. At least 6 of the 8 items on the primary outcome (FACQ Strain subscale at 3 months) had to be completed for inclusion in primary analysis. Similar analysis was used for all numerical secondary outcome measures. Sensitivity analyses were prespecified in the Statistical Analysis Plan to explore any potential bias and examine the robustness of findings.

An analysis plan for the economic evaluation was also published as part of the study protocol. The economic evaluation compared the intervention with usual care over the 6-month follow-up period using an ITT approach and from the NHS and social care perspective. The measure of health benefit was utility, derived from EQ-5D-5L at each assessment using the crosswalk methods as currently recommended by the National Institute for Health and Care Excellence. ${ }^{26}$ Quality-adjusted life years (QALYs) were calculated from these utility values using an area under the curve approach. The costs for the economic evaluation include the costs associated with NHS and social care resources used by carers during the study and the direct costs associated with delivering the intervention/control. The intervention-related costs included training for staff and time spent providing support (extracted from service delivery records). Further details of the economic methods are reported in online supplemental material.

Regression models, based on multiple imputed datasets, were used to estimate net costs (generalised linear model with gamma family and log link) and QALYs 
(linear model) for the intervention arm compared with the control arm. Models allowed for clustering by adjusting for the same cluster-level covariates as the clinical-effectiveness analysis (see above) and the models were specified so that the CIs allowed for intragroup correlation. Net costs were divided by net QALYs to calculate an Incremental Cost-Effectiveness Ratio (ICER). The net costs and QALYs were bootstrapped 2000 times to estimate robust $95 \%$ CIs and plotted on a cost-effectiveness plane. Prespecified sensitivity analyses were conducted, including complete case analyses.

\section{Role of the funding source}

The NIHR CLAHRC had no role in study design, data collection, data analysis, data interpretation or writing of the paper. Stroke Association partnered with NIHR in funding this study and was the specialist stroke service provider in OSCARSS. They did provide some data (eg, service delivery records) and contributed to discussions about data interpretation and dissemination of findings. The corresponding author had full access to all the study data and had final responsibility for the decision to submit for publication.

\section{RESULTS}

In September 2016 we randomised 36 clusters (18 intervention; 18 control). Three control and one intervention cluster withdrew soon after due to decommissioning or all staff long-term sickness (see figure 2) so 32 clusters were trained in January 2017. Three replacement clusters were recruited, randomised and trained between February and April 2017 (one intervention; two control). This gave a total of 35 recruiting clusters (18 intervention; 17 control). Cluster and staff baseline characteristics are included in online supplemental table S2.

Between January 2017 and July 2018, 628 eligible carers (334 intervention; 294 control) were referred for potential participation across 35 participating clusters (18 intervention; 17 control) in England and Northern Ireland. Of those eligible, $414(66 \%)$ carers consented (208 intervention; 206 control) and were followed up between March

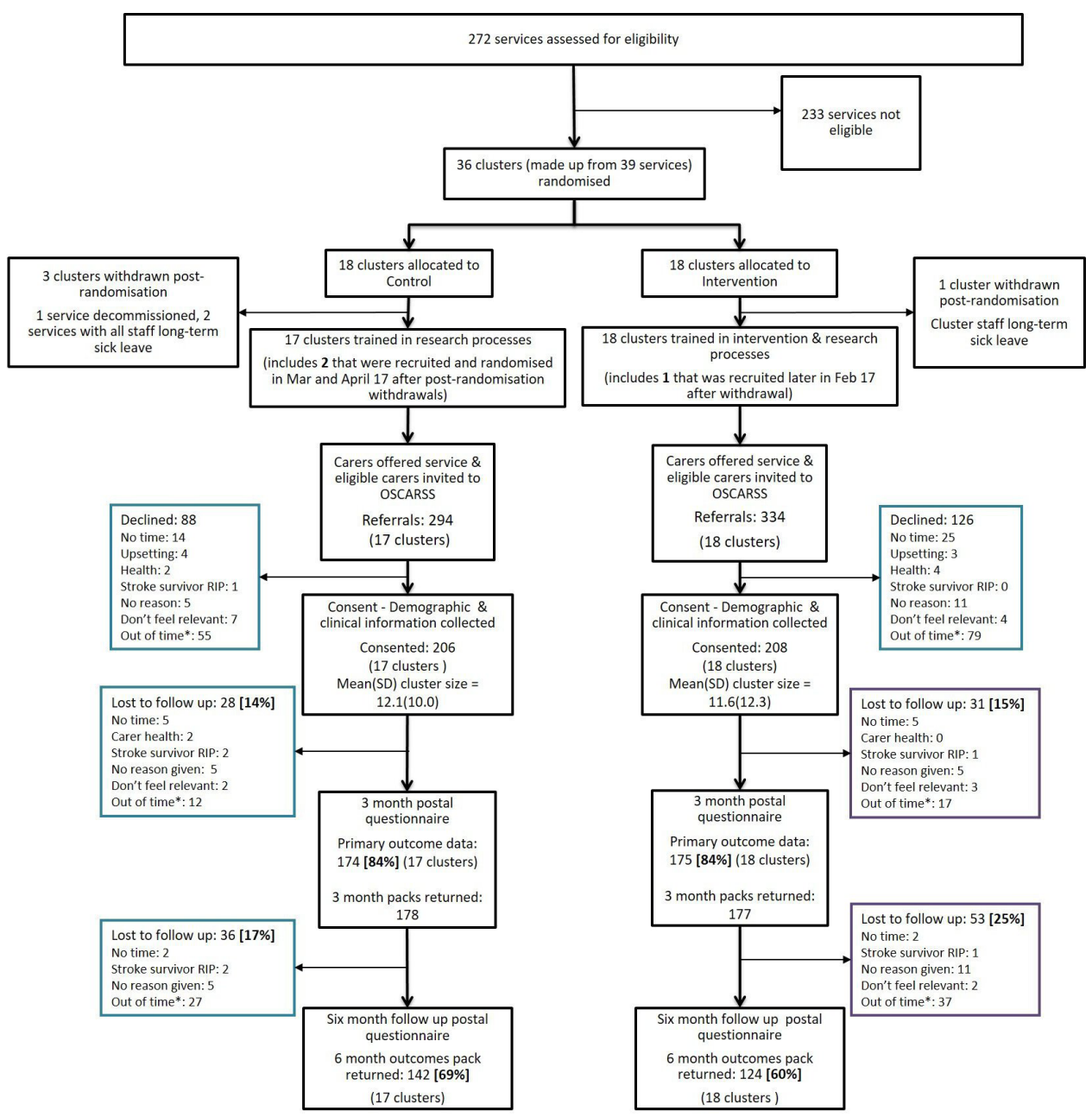

Figure 2 CONSORT diagram showing cluster recruitment and patient flow. All numbers correspond to number of carers unless otherwise stated. All percentages are out of number of consented carers. ${ }^{*}$ Postal packs had not been returned after 13 weeks/21 weeks/26 weeks for demographic/3-month/6-month data. CONSORT, Consolidated Standards of Reporting Trials; OSCARSS, Organising Support for Carers of Stroke Survivors. 
2017 and December 2018. Participant flow is shown in figure 2 and consented carer study entry characteristics are shown in table 2.

Of the 414 consented participants, $319(77 \%)$ were women and $315(76 \%)$ were partners/spouses of the stroke survivor they cared for and $399(97 \%)$ were ethnically white. The mean age of carers was 62 years old when they joined the study and the median time from the stroke event to support being initiated was 2.3 months across the whole sample (IQR=1.1-2.3). All measured variables related to consented carers were well balanced across intervention and control groups, including the level of independence of the cared-for stroke survivor, as perceived by carers.

Primary outcomes were available for $175(84 \%)$ of consented carers in the intervention group and 174 (84\%) in the control group. Follow-up (secondary) outcomes were available for $124(60 \%)$ of consented carers in the intervention group and $142(69 \%)$ in the control group.

Primary analysis of all outcomes is shown in table 3 . Clustering for the primary outcome was low $(\mathrm{ICC}=0.02)$ and negligible after adjustment for covariates. For our primary outcome measure we found the mean (SD) FACQ carer strain at 3 months to be $3.11(0.87)$ in the control group compared with $3.03(0.90)$ in the intervention group, adjusted mean difference -0.04 (95\% CI -0.20 to 0.13 ). Note that this CI excludes the minimal important difference of 0.31 used in our sample size calculation and therefore the data are not consistent with a clinically relevant difference between intervention and control groups. Similarly when we looked at the longer term FACQ carer strain at 6 months we observed a mean control measure of $3.10(0.88)$ compared with 3.07 (0.87), adjusted mean difference -0.04 (95\% CI -0.22 to 0.14 ). All other secondary outcome measures had small differences and tight CIs (see table 3) and therefore are not consistent with meaningful differences between control and intervention. Both unadjusted and adjusted estimates of intervention effect were similar, providing no evidence of any confounding due to demographic or clinical variables. These findings were consistent across all sensitivity analyses including: excluding delayed responders; removing carer dyads; imputing missing outcome data; and combining 3-month and 6-month data, suggesting that the results are robust to assumptions made in the analysis.

The clinical interpretation of selected findings was that for the primary outcome, carer strain, both groups reported an average of around 3 out of 5 that is, a neutral level. For secondary outcomes, average levels of anxiety and depression were around 8 and $6 / 7$ out of 21 (mild and non-case, respectively). Both groups tended to 'agree' with the positive appraisal of the impact of caregiving that is, average scores 4 out of 5 . Satisfaction ratings for both groups were towards the higher end of the composite scale, an average of around 30 out of 44 .

For the economic evaluation, there was a high proportion of missing data but economic analysis was still

\begin{tabular}{|c|c|c|}
\hline & Control & Intervention \\
\hline & $\mathrm{N}=206$ & $\mathrm{~N}=\mathbf{2 0 8}$ \\
\hline \multicolumn{3}{|l|}{ Sex, n (\%) } \\
\hline Male & $42(20.4)$ & $51(24.5)$ \\
\hline Female & $164(79.6)$ & $155(74.5)$ \\
\hline Missing data & - & $2(1)$ \\
\hline Age, mean (range) & $62.5(24-86)$ & $62.3(21-88)$ \\
\hline \multicolumn{3}{|c|}{ Relationship with stroke survivor, n (\%) } \\
\hline $\begin{array}{l}\text { Husband/wife or } \\
\text { partner }\end{array}$ & $160(77.7)$ & $155(74.5)$ \\
\hline Parent & $2(1.0)$ & $6(2.9)$ \\
\hline Son/daughter & $39(18.9)$ & $41(19.7)$ \\
\hline Other & $5(2.5)$ & $5(2.5)$ \\
\hline Missing data & - & $1(0.5)$ \\
\hline \multicolumn{3}{|c|}{ Lives relative to stroke survivor, $\mathrm{n}(\%)$} \\
\hline In the same household & 179 (86.9) & $172(82.7)$ \\
\hline $\begin{array}{l}\text { Within walking } \\
\text { distance }\end{array}$ & $8(3.9)$ & $12(5.8)$ \\
\hline $\begin{array}{l}\text { Within } 30 \text { min drive/ } \\
\text { public transport }\end{array}$ & $16(7.8)$ & $16(7.7)$ \\
\hline $\begin{array}{l}\text { More than } 30 \mathrm{~min} \\
\text { drive/public transport }\end{array}$ & $3(1.5)$ & $8(3.8)$ \\
\hline \multicolumn{3}{|l|}{ Marital status, $\mathrm{n}(\%)$} \\
\hline Single & $18(9)$ & $9(4)$ \\
\hline $\begin{array}{l}\text { Married/living as } \\
\text { married }\end{array}$ & $177(85)$ & $178(87)$ \\
\hline Other & $13(6)$ & $18(8)$ \\
\hline Missing data & $1(0)$ & - \\
\hline \multicolumn{3}{|l|}{ Ethnicity, n (\%) } \\
\hline White & $200(97.1)$ & $199(96.7)$ \\
\hline $\begin{array}{l}\text { Mixed/multiple ethnic } \\
\text { groups }\end{array}$ & - & $4(1.9)$ \\
\hline Asian/Asian British & $6(2.9)$ & $5(2.4)$ \\
\hline \multicolumn{3}{|l|}{ Employment status, n (\%) } \\
\hline Employed full-time & $30(14.6)$ & $25(12.0)$ \\
\hline Employed part-time & $23(11.2)$ & $25(12.0)$ \\
\hline Self-employed & $13(6.3)$ & $9(4.3)$ \\
\hline Retired & $102(49.5)$ & $111(53.4)$ \\
\hline Unemployed & $11(5.3)$ & $12(5.8)$ \\
\hline Full-time education & - & $1(0.5)$ \\
\hline $\begin{array}{l}\text { Other, including } \\
\text { homemaker }\end{array}$ & $27(13.1)$ & $25(12)$ \\
\hline \multicolumn{3}{|c|}{ Highest level of education, n (\%) } \\
\hline None & $47(22.8)$ & $49(23.6)$ \\
\hline Examinations at 16 & $72(35.0)$ & $75(36.1)$ \\
\hline $\begin{array}{l}\text { A/AS level or } \\
\text { equivalent }\end{array}$ & $41(19.9)$ & $29(13.9)$ \\
\hline University & $42(20.4)$ & $50(24.0)$ \\
\hline
\end{tabular}


Table 2 Continued

\begin{tabular}{|ccc|}
\hline & \multicolumn{1}{c}{ Control } & \multicolumn{1}{c}{ Intervention } \\
\cline { 2 - 2 } & $1(0.5)$ & N=208 \\
\hline Other & $3(1.5)$ & $3(1.0)$ \\
\hline Missing data & $124(60.2)$ & $130(62.5)$ \\
\hline Carer has long-term health condition, $\mathrm{n}(\%)$ & \\
\hline Yes & $82(39.8)$ & $78(37.5)$ \\
\hline No &
\end{tabular}

Carer provided care to stroke survivor prior to stroke, $\mathrm{n}(\%)$

$\begin{array}{lrr}\text { Yes } & 81(39.3) & 79(38.0) \\ \text { No } & 124(60.2) & 122(58.7)\end{array}$

Cared-for stroke survivor characteristics (as reported by carer)

Months post-stroke (at date seen)

\begin{tabular}{|lcc|}
\hline Mean (SD) & $5.93(15.47)$ & $6.46(16.38)$ \\
\hline Median (IQR) & $2.2(1.1-4.6)$ & $2.37(1.2-4.8)$ \\
\hline Missing data, n (\%) & $3(1.5)$ & $8(3.8)$ \\
\hline Independence* & & \\
\hline Mean (SD) & $10.99(3.67)$ & $11.14(3.69)$ \\
\hline Median (IQR) & $11(8-14)$ & $11(8-14)$ \\
\hline
\end{tabular}

*Mean score for carer perceived independence calculated over 6 domains: personal care, toilet, cooking, walking, transport and finances/legal issues. Each domain scored 1-3 (total max score $=18$ ) with low scores equating to greater independence.

feasible. We found similar neutral findings between groups in terms of health benefits (see table 4). Resource use is summarised in online supplemental tables S3-S8. Costs associated with the intervention were slightly higher (around £40 per person) than the control, primarily due to:

- Additional staff training required for the intervention, calculated at $£ 15$ per consented carer supported in intervention-allocated clusters.

- Additional support provided to consented carers in intervention-allocated clusters, according to extracted service delivery records. Carers in interventionallocated versus control-allocated clusters had 15 vs 12 support activities recorded, on average, totalling 4.7 hours vs 4.2 hours, respectively.

- Carers in intervention-allocated clusters self-reported accessing more primary care services, specifically general practice nurses.

These slightly higher costs without measurable health benefits over usual care suggest that the intervention as delivered is unlikely to be cost-effective (see table 5). This remained the case in all sensitivity analyses. Figure 3 shows the cost-effectiveness plane for the primary analysis; the clustering around the vertical axis demonstrates that we can be relatively certain there is no additional health benefit from the intervention compared with the control group.
No SAEs were reported that were judged to be related to the research. There were 12 SAEs in total (seven intervention; five control). Ten involved hospitalisation and two related to Accident and Emergency visits with possible long-term incapacity.

Service delivery records indicate that more carers received an individual case record in the intervention arm $(92 / 208,44 \%)$ than control arm $(65 / 206,32 \%)$; other carers had service delivery data captured alongside a stroke survivor record. In addition, intervention arm carers versus control arm carers had more needs reported (146 vs 80 ) and more actions agreed (278 vs $148)$, according to service delivery records.

Indicative findings from the quantitative data on the implementation of the intervention suggest the intervention was not implemented as intended. Overall, of the 334 eligible carers referred to the study from intervention-allocated clusters, the CSNAT-Stroke needs assessment tool and action plan were recorded as used in $278(83 \%)$ and $121(36 \%)$ cases, respectively. Similarly, for the 208/334 carers who went on to join the study from intervention-allocated clusters, they were used in 172 $(83 \%)$ and $66(32 \%)$ cases, respectively.

\section{DISCUSSION}

In terms of clinical effectiveness and cost-effectiveness OSCARSS' findings were conclusive. We found no meaningful difference in the level of self-reported caregiver strain between those allocated to an adapted support intervention or to usual care. Findings were robust and consistent across all outcomes, time points and sensitivity analyses. The economic evaluation demonstrated neutral findings on health benefits and slightly increased costs making the intervention unlikely to be cost-effective compared with usual care. There are several possible explanations for our neutral finding explored in detail below. In brief, carers in both groups received support from the same national service provider organisation, and at the primary outcome time point both groups had a level of strain categorised as neutral on average. Carers seen by intervention-allocated clusters received slightly more support and accessed more primary care services than carers in the control group. However, the intervention was not fully delivered as intended.

\section{Comparison with other studies}

A review of multifaceted support interventions for stroke survivors and carers found no evidence of effectiveness for carers' subjective health status nor mental health (15 interventions, 1775 carers).${ }^{14}$ A review of nonpharmacological interventions for carers of stroke survivors also found no strong evidence to inform best practice for supporting carers (8 studies, 1007 carers).${ }^{13}$ Recent important randomised trials of structured training for carers to provide care $^{11}$ or deliver rehabilitation ${ }^{12}$ show the feasibility of carer trials but an absence of evidence of effectiveness. 
Table 3 Primary analysis of all outcomes

\begin{tabular}{|c|c|c|c|}
\hline & Control & Intervention & \multirow{2}{*}{$\begin{array}{l}\text { Difference }(95 \% \mathrm{Cl}) \\
\text { Adjusted for } \\
\text { clustering and } \\
\text { demographic } \\
\text { variables }\end{array}$} \\
\hline & Mean (SD) & Mean (SD) & \\
\hline \multirow[t]{2}{*}{ Primary outcome: FACQ carer strain at 3 months } & $\mathrm{N}=174$ & $\mathrm{~N}=175$ & $-0.04(-0.20$ to 0.13$)$ \\
\hline & $3.11(0.87)$ & $3.03(0.90)$ & \\
\hline \multicolumn{4}{|c|}{ Secondary outcomes collected at 3 months after support initiated: } \\
\hline \multirow[t]{2}{*}{ FACQ carer distress } & $\mathrm{N}=173$ & $\mathrm{~N}=176$ & $0.04(-0.13$ to 0.21$)$ \\
\hline & $2.88(0.83)$ & $2.91(0.85)$ & \\
\hline \multirow[t]{2}{*}{ FACQ positive caregiving appraisal } & $\mathrm{N}=175$ & $\mathrm{~N}=176$ & $0.05(-0.06$ to 0.17$)$ \\
\hline & $3.99(0.61)$ & $4.05(0.54)$ & \\
\hline \multirow[t]{2}{*}{ Pound Satisfaction with stroke services (composite) } & $\mathrm{N}=177$ & $\mathrm{~N}=171$ & $-1.06(-3.35$ to 1.23$)$ \\
\hline & $31.14(8.85)$ & $30.51(10.36)$ & \\
\hline \multirow[t]{2}{*}{ Pound overall Satisfaction with stroke services (smiley faces) } & $\mathrm{N}=174$ & $\mathrm{~N}=167$ & $0.00(-0.30$ to 0.31$)$ \\
\hline & $5.10(1.51)$ & $5.10(1.49)$ & \\
\hline \multirow[t]{2}{*}{ HADS anxiety } & $\mathrm{N}=174$ & $\mathrm{~N}=172$ & $0.04(-0.89$ to 0.97$)$ \\
\hline & $8.34(4.51)$ & $8.20(4.73)$ & \\
\hline \multirow[t]{2}{*}{ HADS depression } & $\mathrm{N}=174$ & $\mathrm{~N}=172$ & $-0.06(-0.86$ to 0.73$)$ \\
\hline & $6.30(4.17)$ & $6.12(4.07)$ & \\
\hline \multicolumn{4}{|c|}{ Follow-up outcomes collected at 6 months after support initiated: } \\
\hline \multirow[t]{2}{*}{ FACQ carer strain } & $\mathrm{N}=140$ & $\mathrm{~N}=121$ & $-0.04(-0.22$ to 0.14$)$ \\
\hline & $3.10(0.88)$ & $3.07(0.87)$ & \\
\hline \multirow[t]{2}{*}{ FACQ carer distress } & $\mathrm{N}=140$ & $\mathrm{~N}=121$ & $-0.03(-0.23$ to 0.16$)$ \\
\hline & $2.93(0.84)$ & $2.92(0.84)$ & \\
\hline \multirow[t]{2}{*}{ FACQ positive caregiving appraisal } & $N=140$ & $\mathrm{~N}=121$ & $0.12(-0.02$ to 0.26$)$ \\
\hline & $3.91(0.64)$ & $4.04(0.54)$ & \\
\hline \multirow[t]{2}{*}{ Pound Satisfaction with stroke services (composite) } & $\mathrm{N}=136$ & $\mathrm{~N}=121$ & $-1.48(-3.40$ to 0.44$)$ \\
\hline & $32.12(5.88)$ & $30.58(9.81)$ & \\
\hline \multirow[t]{2}{*}{ Pound overall Satisfaction with stroke services (smiley faces) } & $\mathrm{N}=138$ & $\mathrm{~N}=120$ & $-0.21(-0.61$ to 0.20$)$ \\
\hline & $5.17(1.51)$ & $4.99(1.54)$ & \\
\hline \multirow[t]{2}{*}{ HADS anxiety } & $\mathrm{N}=141$ & $\mathrm{~N}=123$ & $0.13(-0.98$ to 1.23$)$ \\
\hline & $8.90(4.66)$ & $8.95(5.10)$ & \\
\hline \multirow[t]{2}{*}{ HADS depression } & $N=141$ & $\mathrm{~N}=123$ & $-0.43(-1.36$ to 0.51$)$ \\
\hline & $7.06(4.56)$ & $6.65(4.06)$ & \\
\hline
\end{tabular}

FACQ, Family Appraisal of Caregiving Questionnaire; HADS, Hospital Anxiety and Depression Scale.

Prior to OSCARSS there was no robust RCT evidence of the CSNAT, or any other approach, to guide the support of carers of stroke survivors. A non-randomised study of CSNAT with a non-stroke population concluded that the CSNAT was associated with small to moderate reductions in carer strain compared with pre-intervention. ${ }^{68}$ Several UK studies by the CSNAT team showed similar outcomes and good acceptability, but also reported implementation challenges similar to those found in OSCARSS $^{57}$ and discussed in our sister process evaluation paper. ${ }^{17}$

\section{Strengths and limitations, with consideration of clinical} implications

To understand the clinical implications of these findings, we consider the study's strengths and limitations, and explore possible explanatory factors: the choice of comparator; intervention delivered; the timing and choice of outcomes and characteristics of the sample.

Features of the study design and conduct ensured good internal validity. For example, clusters were recruited prior to stratified randomisation and carer research participants-who completed self-reported outcome 
Table 4 EQ-5D utility values at each time point and QALYs for whole follow-up, by treatment arm

\begin{tabular}{|c|c|c|}
\hline & Control & Intervention \\
\hline & \multicolumn{2}{|l|}{ Mean $(95 \% \mathrm{Cl})$} \\
\hline \multirow[t]{2}{*}{ Study entry utility } & 0.78 (0.75 to 0.81$)$ & 0.76 (0.74 to 0.79$)$ \\
\hline & $\mathrm{n}=204$ & $n=199$ \\
\hline \multirow[t]{2}{*}{ 3-month utility } & 0.73 (0.71 to 0.76$)$ & $0.73(0.70$ to 0.76$)$ \\
\hline & $\mathrm{n}=177$ & $n=165$ \\
\hline \multirow[t]{2}{*}{ 6-month utility } & $0.72(0.69$ to 0.75$)$ & $0.73(0.69$ to 0.76$)$ \\
\hline & $n=136$ & $\mathrm{n}=118$ \\
\hline \multirow{2}{*}{$\begin{array}{l}\text { QALYs (over } 6 \\
\text { months) }\end{array}$} & $0.37(0.36$ to 0.38$)$ & $0.38(0.36$ to 0.39$)$ \\
\hline & $n=135$ & $n=103$ \\
\hline Net QALYs* & \multicolumn{2}{|c|}{$0.009(-0.016$ to 0.033$) n=238$} \\
\hline $\begin{array}{l}\text { Adjusted net } \\
\text { QALYs } †\end{array}$ & \multicolumn{2}{|c|}{$0.004(-0.018$ to 0.026$) n=227$} \\
\hline
\end{tabular}

*Unadjusted but allowing for intracluster correlation in SEs. †Net QALYs calculated using linear regression model adjusted for age, time since stroke, stroke severity, whether or not the carer had any long-term health conditions, cluster size and years of experience of the cluster staff.

QALYs, quality-adjusted life years.

measures-were unaware of allocation. Cluster trials risk imbalance across trial groups ${ }^{27}$ but in OSCARSS, steps were taken to minimise this and all measured variables related to consented carers appeared well-balanced across arms. The intervention was implemented at cluster level and began the moment a carer came into

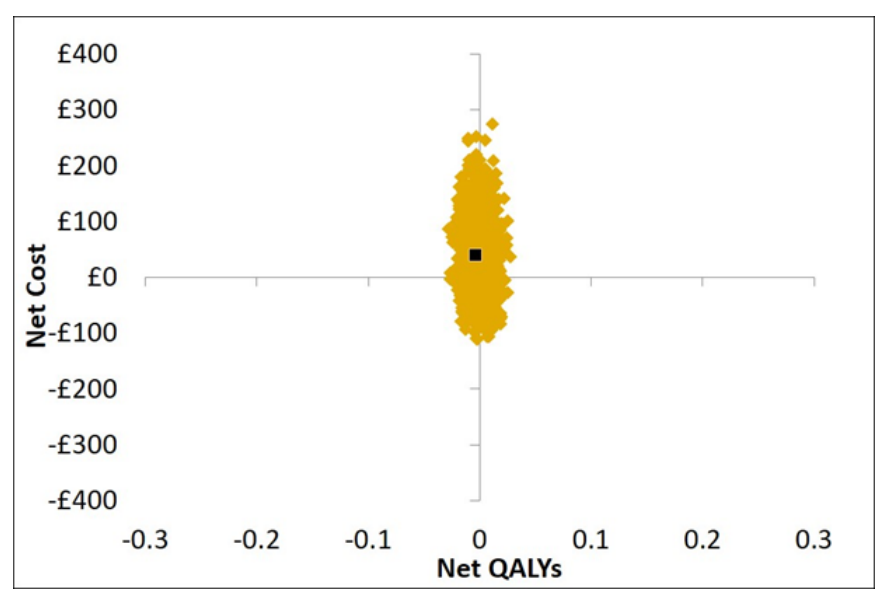

Figure 3 Cost-effectiveness plane for primary analysis. The cost-effectiveness plane shows the ICER (large square) and 2000 bootstrapped estimates of net costs and QALYs. The narrow, even, horizontal spread of the points indicates low uncertainty regarding the indifferent health benefit. The broader vertical spread of the points shows that there is more uncertainty around the costs. ICER, incremental costeffectiveness ratio; QALYs, quality-adjusted life years.

contact with the service provider so it was not possible to explore change from baseline in individual outcomes, however the randomised design, coupled with balanced cluster and carer characteristics, helps overcome this. OSCARSS achieved its target, powered sample size with minimal missing clinical data and low attrition $(16 \%$ in both groups) at the primary outcome time point. We have confidence in our findings which were consistent across

Table 5 Results of primary and sensitivity economic analyses comparing CSNAT intervention with usual care

\begin{tabular}{|c|c|c|c|}
\hline & Net costs $(95 \% \mathrm{Cl})$ & Net QALYs (95\% CI) & ICER (£/QALY) \\
\hline \multicolumn{4}{|l|}{ Primary analysis } \\
\hline $\begin{array}{l}\text { Multiple imputed datasets } \\
(\mathrm{n}=410)^{\star}\end{array}$ & $£ 39.05$ (-69.61 to 147.71$)$ & $-0.004(-0.020$ to 0.012$)$ & Intervention is dominated \\
\hline \multicolumn{4}{|l|}{ Sensitivity analyses } \\
\hline Complete cases $(n=131)$ & $£ 41.24(-29.01$ to 111.49$)$ & $-0.0001(-0.026$ to 0.026$)$ & Intervention is dominated \\
\hline $\begin{array}{l}\text { Exclude training and } \\
\text { intervention costs }(n=410)^{\star}\end{array}$ & $£ 23.33(-98.21$ to 144.87$)$ & $-0.004(-0.020$ to 0.012$)$ & Intervention is dominated \\
\hline \multicolumn{4}{|l|}{ Alternative outcome measure } \\
\hline & Net costs $(95 \% \mathrm{Cl})$ & Net change $(95 \% \mathrm{Cl})$ & $\begin{array}{l}\text { ICER: (£/1 point } \\
\text { improvement) }\end{array}$ \\
\hline
\end{tabular}

All analyses adjusted for covariates: carer's age, time since stroke, stroke severity, whether or not carer has long-term health conditions, length of experience of cluster staff, size of cluster and cluster ID.

Cls for all analyses calculated following bootstrapping: 2000 times for imputed datasets, 10000 times for complete case datasets.

*Four participants with no baseline EQ-5D data were excluded from the imputation, leaving 410 participants.

†Thirty-six participants in the imputed dataset excluded who violated protocol conditions (multiple carers per stroke survivor or questionnaires returned late).

CSNAT, Carer Support Needs Assessment Tool; FACQ, Family Appraisal of Caregiving Questionnaire; ICER, incremental cost-effectiveness ratio; QALYs, quality-adjusted life years. 
all sensitivity analyses including for protocol deviations such as the late return of postal questionnaires.

The demographic profile of the sample was as expected for carers of stroke survivors and in keeping with other trials. ${ }^{11}$ However, as is so often the case in UK-based stroke trials, the sample lacked ethnic diversity $(<3 \%$ non-white group). This does not reflect the diversity in the UK general population. Stroke trials need strategies to achieve equity of access, given that a large portion of UK stroke admissions are from Black, Asian and minority ethnic communities. ${ }^{28}$ We aimed to recruit the primary caregiver but did not collect additional data on whether they were caring alone or with support. All other measured carer variables were balanced across randomised groups.

Neutral findings must consider the context that carers in both groups received support, from the same stroke specialist provider organisation, and reported high satisfaction with stroke services on average. This and the outcomes achieved suggest it is plausible that both methods of support delivered in OSCARSS were beneficial to carers.

We collaborated closely with our service provider to pragmatically tailor the intervention for implementation, which improved buy-in by the organisation and cluster staff. However, our data show that the intervention's assessment tool and action plan were underused. Implementation was explored in greater depth in the embedded process evaluation and is consistent with these quantitative indicators; namely, that the intervention as intended was not fully implemented. ${ }^{17}$

We have no data beyond 6 months after support had been initiated, and while our inclusion criteria aimed to recruit carers at varying stages, our sample was predominantly early post-stroke. Previous stroke research suggests caregivers may take months to adjust to their role as caregivers, become aware of and prioritise their own needs. ${ }^{29}$ The OSCARSS process evaluation and opinions of members of our study-specific carer advisory research group endorse this and suggest that, while informal caregivers need support early after stroke, they may struggle to participate fully in a 'carer-led' intervention that encourages self-management, such as the CSNAT intervention, which could have contributed to the implementation issues noted above. In addition, our relatively short follow-up period of 6 months may have been too early to detect any impact of carers in the intervention group receiving more support and accessing more primary healthcare services, as observed in our economic evaluation. While our choice of primary outcome was informed by past research using the CSNAT intervention ${ }^{68}$ and the preferences of our service user group of stroke carers, our measure may not have been adequate to detect a difference in our population of stroke carers.

\section{CONCLUSIONS}

In summary, OSCARSS found that the CSNAT-Stroke intervention was not measurably clinically effective or cost-effective compared with usual care from a stroke specialist provider organisation, although we have substantial evidence that the intervention was not fully implemented in this pragmatic trial. OSCARSS demonstrated that methodologically rigorous research evaluations for carers of stroke survivors can be successfully delivered by voluntary sector organisations. However, the challenges of fully implementing person-centred care in research and service development need to be addressed through enhanced and ongoing staff training as well as organisational mechanisms to support and champion new approaches becoming embedded into practice. There remains a high priority for research to determine how best to support carers of stroke survivors.

\section{Author affiliations}

${ }^{1}$ Division of Neuroscience and Experimental Psychology, The University of Manchester, Manchester Academic Health Sciences Centre (MAHSC), Manchester, UK

${ }^{2}$ National Institute for Health Research Collaboration for Leadership in Applied Health Research and Care Greater Manchester (NIHR CLAHRC GM), Manchester, UK ${ }^{3}$ Centre for Biostatistics, Division of Population Health, Health Services Research \& Primary Care, The University of Manchester, Manchester, UK

${ }^{4}$ Manchester Centre for Health Economics, Division of Population Health, Health Services Research \& Primary Care, School of Health Sciences, The University of Manchester, Manchester, UK

${ }^{5}$ Alliance Manchester Business School, The University of Manchester, Manchester, UK

${ }^{6}$ Centre for Reviews and Dissemination, University of York, York, UK

${ }^{7}$ Division of Nursing Midwifery and Social Work, School of Health Sciences, The University of Manchester, Manchester Academic Health Sciences Centre (MAHSC), Manchester, UK

${ }^{8}$ Centre for Family Research, University of Cambridge, Cambridge, UK

Twitter Emma Patchwood @DrPatchwood, Sarah Darley @sardarl, Gunn Grande @gunn_grande, Gail Ewing @gailewing_cfr and Audrey Bowen @ audreybowenprof

Acknowledgements The authors would like to thank all of the carers and staff members who participated and contributed to this study. We would also like to thank and acknowledge members of the supportive NIHR CLARHC GM OSCARSS Research Team who helped make the study possible. This includes members of the OSCARSS carer advisory Research User Group (Kelly Burke, Christine Halford, Natalie Halford, Geoff Heathcote, Kath Purcell and Ben Wright); members from the trial management group (Caroline 0'Donnell (data analyst), Alison Littlewood and Katy Rothwell (programme managers), Sam Wilkinson, Amy Woodhouse and Rose Crees (administrative assistants), Aneela Macavoy and Zoe Ashton (research facilitators)); and members of the Trial Steering Committee (Chris Sutton (chair and statistician), David Clarke (process evaluation steer), Rachael Hunter (health economics steer), Jordi Morell (clinical steer) and Nigel Bamford (lay member)).

Contributors EP and AB are co-chief investigators of OSCARSS and conceived the study. EP wrote the first draft of the paper and all authors substantially contributed to revisions. KW-N project managed OSCARSS. SAR and EB performed statistical analysis. EC performed economic analysis. SK and SD led the process evaluation in OSCARSS and contributed to interpretation of the implementation data presented in this paper. GG and GE developed the original CSNAT intervention and contributed substantially to descriptions of the adapted CSNAT-Stroke intervention in this paper.

Funding This study was funded by the National Institute for Health Research Collaboration for Leadership in Applied Health Research and Care Greater Manchester (NIHR CLAHRC GM), grant number (N/A), partnered with Stroke Association Funding, grant number (N/A).

Competing interests $A B, G G, S A R, E B$ and GE held grants with NHHR during the course of the OSCARSS Study. AB and EP additionally hold grants with Stroke Association outside of this work. There are no other relationships or activities that could appear to have influenced the submitted work.

Patient consent for publication Not required. 
Ethics approval Ethics approvals were obtained from Lancaster Research Ethics Committee (ref: 16/NW/0657).

Provenance and peer review Not commissioned; externally peer reviewed.

Data availability statement Data are available upon reasonable request. Requests for data and statistical code should be made to the corresponding author and will be considered by members of the original trial management group, including the co-chief investigators, who will release data on a case-by-case basis. Data will be shared following the principles for sharing patient-level data as described by Smith et al (2015). The data will not contain any direct identifiers, we will minimise indirect identifiers and remove free text data, to minimise the risk of identification.

Supplemental material This content has been supplied by the author(s). It has not been vetted by BMJ Publishing Group Limited (BMJ) and may not have been peer-reviewed. Any opinions or recommendations discussed are solely those of the author(s) and are not endorsed by BMJ. BMJ disclaims all liability and responsibility arising from any reliance placed on the content. Where the content includes any translated material, BMJ does not warrant the accuracy and reliability of the translations (including but not limited to local regulations, clinical guidelines, terminology, drug names and drug dosages), and is not responsible for any error and/or omissions arising from translation and adaptation or otherwise.

Open access This is an open access article distributed in accordance with the Creative Commons Attribution Non Commercial (CC BY-NC 4.0) license, which permits others to distribute, remix, adapt, build upon this work non-commercially, and license their derivative works on different terms, provided the original work is properly cited, appropriate credit is given, any changes made indicated, and the use is non-commercial. See: http://creativecommons.org/licenses/by-nc/4.0/.

\section{ORCID iDs}

Emma Patchwood http://orcid.org/0000-0002-4198-5761

Elizabeth Camacho http://orcid.org/0000-0001-9574-7710

Sarah Darley http://orcid.org/0000-0001-5420-6774

Gunn Grande http://orcid.org/0000-0003-2200-1680

Gail Ewing http://orcid.org/0000-0001-9547-7247

Audrey Bowen http://orcid.org/0000-0003-4075-1215

\section{REFERENCES}

1 Anderson R. The unremitting burden on carers. BMJ 1987;294:73-4.

2 Carers UK. State of caring report, 2019. Available: www.carersuk.org/ stateofcaring-report

3 Department of Health. Care act, 2014. Available: http://www. legislation.gov.uk/ukpga/2014/23/contents/enacted/data.htm

4 Ewing G, Grande G, National Association for Hospice at Home. Development of a carer support needs assessment tool (CSNAT) for end-of-life care practice at home: a qualitative study. Palliat Med 2013:27:244-56.

5 Aoun S, Deas K, Toye C, et al. Supporting family caregivers to identify their own needs in end-of-life care: qualitative findings from a stepped wedge cluster trial. Palliat Med 2015;29:508-17.

6 Aoun SM, Grande G, Howting D, et al. The impact of the carer support needs assessment tool (CSNAT) in community palliative care using a stepped wedge cluster trial. PLoS One 2015;10:e0123012.

7 Ewing G, Austin L, Grande G. The role of the carer support needs assessment tool in palliative home care: a qualitative study of practitioners' perspectives of its impact and mechanisms of action. Palliat Med 2016;30:392-400.

8 Grande GE, Austin L, Ewing G. Assessing the impact of a carer support needs assessment tool (CSNAT) intervention in palliative home care: a stepped wedge cluster trial. BMJ Support Palliat Care 2017;7:326-34.
9 Cooper B, Kinsella GJ, Picton C. Development and initial validation of a family appraisal of caregiving questionnaire for palliative care. Psychooncology 2006;15:613-22.

10 Adamson J, Beswick A, Ebrahim S. Is stroke the most common cause of disability? J Stroke Cerebrovasc Dis 2004;13:171-7.

11 Forster A, Dickerson J, Young J, et al. A structured training programme for caregivers of inpatients after stroke (TRACS): a cluster randomised controlled trial and cost-effectiveness analysis. Lancet 2013;382:2069-76.

12 Lindley RI, Anderson CS, Billot L, et al. Family-led rehabilitation after stroke in India (attend): a randomised controlled trial. Lancet 2017;390:588-99.

13 Legg LA, Quinn TJ, Mahmood F, et al. Non-pharmacological interventions for caregivers of stroke survivors. Cochrane Database Syst Rev 2011;10:CD008179.

14 Ellis G, Mant J, Langhorne P, et al. Stroke liaison workers for stroke patients and carers: an individual patient data meta-analysis. Cochrane Database Syst Rev 2010;17:CD005066.

15 Cameron JI, Naglie G, Gignac MAM, et al. Randomized clinical trial of the timing it right stroke family support program: research protocol. BMC Health Serv Res 2014;14:18.

16 Mitchell C, Burke K, Halford N, et al. Value and learning from carer involvement in a cluster randomised controlled trial and process evaluation - Organising Support for Carers of Stroke Survivors (OSCARSS). Res Involv Engagem 2020;6.

17 Darley S, Knowles S, Woodward- Nutt K, et al. Challenges implementing a carer support intervention within a national stroke organisation: findings from the process evaluation of the OSCARSS trial. BMJ Open 2020.

18 Patchwood E, Rothwell K, Rhodes S, et al. Organising support for carers of stroke survivors (OSCARSS): study protocol for a cluster randomised controlled trial, including health economic analysis. Trials 2019;20:19

19 Staniszewska S, Brett J, Simera I, et al. GRIPP2 reporting checklists: tools to improve reporting of patient and public involvement in research. Res Involv Engagem 2017;3:13.

20 Hoffmann TC, Glasziou PP, Boutron I, et al. Better reporting of interventions: template for intervention description and replication (TIDieR) checklist and guide. BMJ 2014;348:g1687.

21 Herdman M, Gudex C, Lloyd A, et al. Development and preliminary testing of the new five-level version of EQ-5D (EQ-5D-5L). Qual Life Res 2011;20:1727-36.

22 Pound P, Gompertz P, Ebrahim S. Development and results of a questionnaire to measure carer satisfaction after stroke. J Epidemiol Community Health 1993;47:500-5.

23 Zigmond AS, Snaith RP. The hospital anxiety and depression scale. Acta Psychiatr Scand 1983;67:361-70.

24 Batistatou E, Roberts C, Roberts S. Sample size and power calculations for trials and quasi-experimental studies with clustering. Stata J 2014;14:159-75.

25 Campbell MK, Thomson S, Ramsay CR, et al. Sample size calculator for cluster randomized trials. Comput Biol Med 2004;34:113-25.

26 van Hout B, Janssen MF, Feng Y-S, et al. Interim scoring for the EQ$5 \mathrm{D}-5 \mathrm{~L}$ : mapping the EQ-5D-5L to EQ-5D-3L value sets. Value Health 2012:15:708-15.

27 Farrin A, Russell I, Torgerson D, et al. Differential recruitment in a cluster randomized trial in primary care: the experience of the UK back pain, exercise, active management and manipulation (UK beam) feasibility study. Clin Trials 2005;2:119-24.

28 Stroke Association. State of the nation: stroke statistics. Available: https://www.stroke.org.uk/sites/default/files/state_of_the_nation_ 2017_final_1.pdf2017

29 Cameron JI, Gignac MAM. "Timing It Right": a conceptual framework for addressing the support needs of family caregivers to stroke survivors from the hospital to the home. Patient Educ Couns 2008;70:305-14. 